\title{
The diagnostic value of metagenomic next-generation sequencing in infectious diseases
}

Hongxia Duan ${ }^{1+}$, Xuan $\mathrm{Li}^{1+}$, Aihong Mei ${ }^{1+}$, Ping $\mathrm{Li}^{1}$, Yang $\mathrm{Liu}^{1}$, Xiaofeng $\mathrm{Li}^{2}$, Weiwei $\mathrm{Li}^{3}$, Changhui Wang ${ }^{{ }^{*}}$ (I) and Shuanshuan Xie ${ }^{1 *}$

\begin{abstract}
Background: Although traditional diagnostic techniques of infection are mature and price favorable at present, most of them are time-consuming and with a low positivity. Metagenomic next-generation sequencing (mNGS) was studied widely because of identification and typing of all pathogens not rely on culture and retrieving all DNA without bias. Based on this background, we aim to detect the difference between mNGS and traditional culture method, and to explore the relationship between mNGS results and the severity, prognosis of infectious patients.

Methods: 109 adult patients were enrolled in our study in Shanghai Tenth People's Hospital from October 2018 to December 2019. The diagnostic results, negative predictive values, positive predictive values, false positive rate, false negative rate, pathogen and sample types were analyzed by using both traditional culture and mNGS methods. Then, the samples and clinical information of 93 patients in the infected group (ID) were collected. According to whether mNGS detected pathogens, the patients in ID group were divided into the positive group of 67 cases and the negative group of 26 cases. Peripheral blood leukocytes, C-reactive protein (CRP), procalcitonin (PCT) and neutrophil counts were measured, and the concentrations of IL-2, IL-4, IL-6, TNF- $a, I L-17 A, I L-10$ and INF- $\gamma$ in the serum were determined by ELISA. The correlation between the positive detection of pathogens by mNGS and the severity of illness, hospitalization days, and mortality were analyzed.

(Continued on next page)
\end{abstract}

\footnotetext{
* Correspondence: wang-chang-hui@hotmail.com;

xieshuanshuan@aliyun.com

${ }^{\dagger}$ Hongxia Duan, Xuan Li and Aihong Mei contributed equally to this work.

'Department of Respiratory Medicine, Shanghai 10th People's Hospital,

Tongji University School of Medicine, \#301, Mid Yanchang Rd, Shanghai

200072, China

Full list of author information is available at the end of the article
}

(C) The Author(s). 2021 Open Access This article is licensed under a Creative Commons Attribution 4.0 International License, which permits use, sharing, adaptation, distribution and reproduction in any medium or format, as long as you give appropriate credit to the original author(s) and the source, provide a link to the Creative Commons licence, and indicate if changes were made. The images or other third party material in this article are included in the article's Creative Commons licence, unless indicated otherwise in a credit line to the material. If material is not included in the article's Creative Commons licence and your intended use is not permitted by statutory regulation or exceeds the permitted use, you will need to obtain permission directly from the copyright holder. To view a copy of this licence, visit http://creativecommons.org/licenses/by/4.0/ The Creative Commons Public Domain Dedication waiver (http://creativecommons.org/publicdomain/zero/1.0/) applies to the data made available in this article, unless otherwise stated in a credit line to the data. 


\begin{abstract}
(Continued from previous page)
Results: 109 samples were assigned into infected group (ID, 92/109, 84.4\%), non-infected group (NID, 16/109, $14.7 \%)$, and unknown group (1/109, 0.9\%). Blood was the most abundant type of samples with 37 cases, followed by bronchoalveolar lavage fluid in 36 cases, tissue, sputum, pleural effusion, cerebrospinal fluid (CSF), pus, bone marrow and nasal swab. In the ID group, the majority of patients were diagnosed with lower respiratory system infections $(73 / 109,67 \%)$, followed by bloodstream infections, pleural effusion and central nervous system infections. The sensitivity of mNGS was significantly higher than that of culture method (67.4\% vs $23.6 \%$; $P<0.001)$, especially in sample types of bronchoalveolar lavage fluid $(P=0.002)$, blood $(P<0.001)$ and sputum $(P=0.037)$, while the specificity of mNGS was not significantly different from culture method ( $68.8 \%$ vs $81.3 \% ; P=0.41)$. The number of hospitals stays and 28-day-motality in the positive mNGS group were significantly higher than those in the negative group, and the difference was statistically significant $(P<0.05)$. Age was significant in multivariate logistic analyses of positive results of mNGS.

Conclusions: The study found that mNGS had a higher sensitivity than the traditional method, especially in blood, bronchoalveolar lavage fluid and sputum samples. And positive mNGS group had a higher hospital stay, 28-daymortality, which means the positive of pathogen nucleic acid sequences detection may be a potential high-risk factor for poor prognosis of adult patients and has significant clinical value. MNGS should be used more in early pathogen diagnosis in the future.
\end{abstract}

Keywords: Next-generation sequencing, Sensitivity, Diagnostic, Infection, Survival

\section{Background}

Infectious diseases are a leading cause of morbidity and mortality worldwide and spread quickly. As the first-line of pathogen detection, microbiology laboratory plays an important role in infection control by means of microscopic examination, culture, identification, drug sensitivity and so on [1]. However, the limitation of molecular diagnosis and genotyping methods remain that pathogens are undetected in up to $60 \%$ of cases [2-4]. Failure to identify pathogens in time may delay the precise treatment of antibiotics, leading to unnecessary use of broad-spectrum antibiotics, inducing resistance, and increasing medical costs [5].

With the completion of the human genome project in the early twenty-first century and the rapid development of sequencing technology, high-throughput and low-cost second-generation sequencing technology emerged [6]. It had been used in whole genome sequencing, whole exome sequencing, macro gene sequencing and so on, among which metagenomic next-generation sequencing (mNGS) was studied most widely. The advantage of mNGS lies in the single run to obtain the sequence information of microbial nucleic acid fragments, through analysis and comparison of which to detect all microbial species and sequence [7]. Besides, mNGS can be used for the identification and typing of all pathogens because mNGS does not rely on culture and retrieve all DNA without bias [8]. Based on mNGS results, antimicrobial resistance, virulence, typing and other information can be used for epidemic investigation. It lays a theoretical foundation for the investigation of infectious diseases outbreak in hospital. Therefore, this technology may play a huge role in infection prevention and medical microbiology laboratory.
Thus, based on microbiome sequencing technology, we compared the sensitivity and specificity of mNGS method and traditional culture method to detect pathogens, and discussed the influence of mNGS detection results on the severity and prognosis of patients with infection in our study.

\section{Methods \\ Study patients}

We retrospectively reviewed 161 cases suspected of acute or chronic infection from respiratory and critical care medicine department, geriatric department, emergency intensive care unit and emergency department at Shanghai 10th People's Hospital in Shanghai, China, between October 2018 and December 2019. Excluding patients with pregnancy, mental illness and under the age of 18, 109 samples were included in our study and for analysis and then they were categorized into 3 groups, infectious disease (ID) group, noninfectious disease (NID) group, and unknown group according to final diagnosis. Specimens were subjected to mNGS testing (BGI, Intertek, Biotecan, China) and regular clinical microbiological assay in a pairwise manner and final diagnosis was determined by clinicians based on both of them and imaging, clinical feature of patients. Meanwhile, clinical data of all enrolled patients, including complete blood count, C-reactive protein (CRP), procalcitonin (PCT), neutrophil count, interleukin (IL)-2, IL-4, IL-6, Tumor Necrosis Factor- $\alpha$ (TNF- $\alpha$ ), IL-17A, IL-10 and Interferon- $\gamma$ (INF- $\gamma$ ) were collected. The flow diagram of cases inclusion and exclusion was shown in Fig. 1. This research had been approved by the ethics 


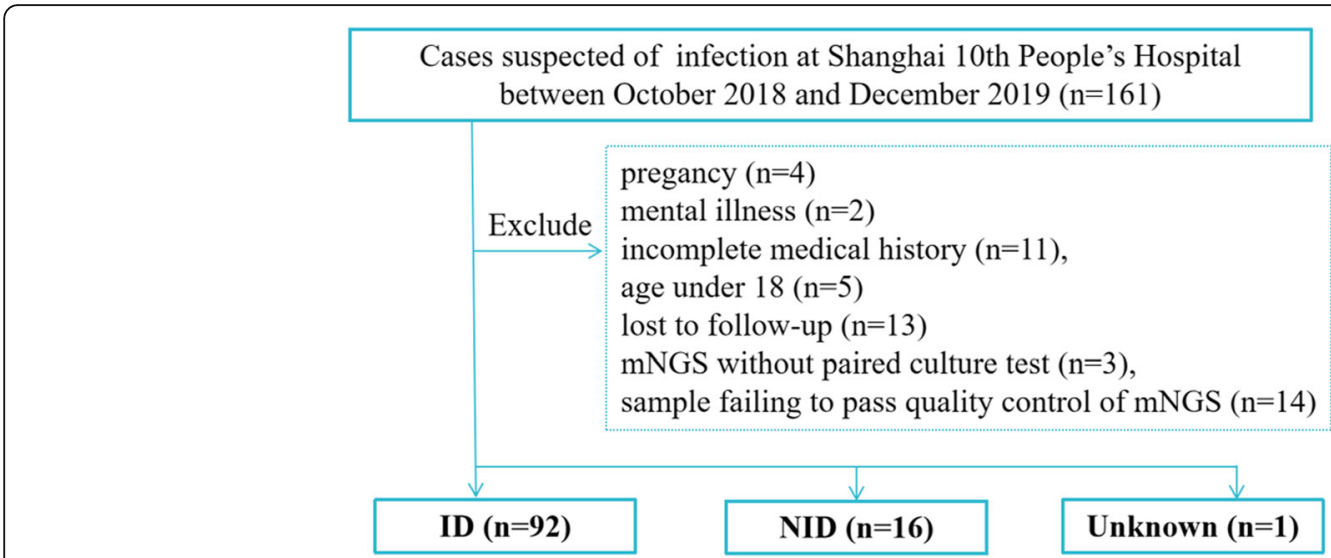

Fig. 1 Flow diagram of cases inclusion and exclusion

committee of the 10th People's Hospital affiliated to Tongji University (No. SHSY-IEC-4.1/20-21/01).

\section{Metagenomic next-generation sequencing and analysis} Nucleic acid detection and sequencing were performed based on BGISEQ-50 platform (BGI-Tianjin, Tianjin, China) in this research. After the sample was taken, nucleic acid was extracted, the library was built and sequenced, and finally the data was analyzed by using the microbiome database (ftp://ftp.ncbi.nlm.nih.gov/genomes/ ). The experimental process was shown in Fig. 2.

\section{Sample processing and library construction (Fig. 2a)}

For infected patients or patients with fever of unknown cause, infected site samples or blood were collected according to standard procedures. Each blood, bronchoalveolar lavage Fluid (BALF) or urine sample was at least $5 \mathrm{ml}(\mathrm{ml})$ and at least $3 \mathrm{ml}$ of each sample of cerebrospinal fluid, sputum, or other sterile liquid. Blood must be collected in anticoagulant tube and stored at room temperature, the protective agent in anticoagulant tube is Ethylene Diamine Tetraacetic Acid (EDTA) anticoagulant and special deoxyribonucleic acid (DNA) protective agent. Other samples were collected in sterile tube and stored at $-80^{\circ} \mathrm{C}$. Blood was transported at room temperature, and other sterile samples were transported in drikold. Since most of the collected samples contain pathogenic pathogens, they were inactivated $\left(56^{\circ} \mathrm{C}, \quad 30 \mathrm{~min}\right)$ before nucleic acid extraction. In addition to this, blood samples were centrifuged to separate plasma and leukocytes when intracellular bacterial infection was particularly suspicious by clinicians.

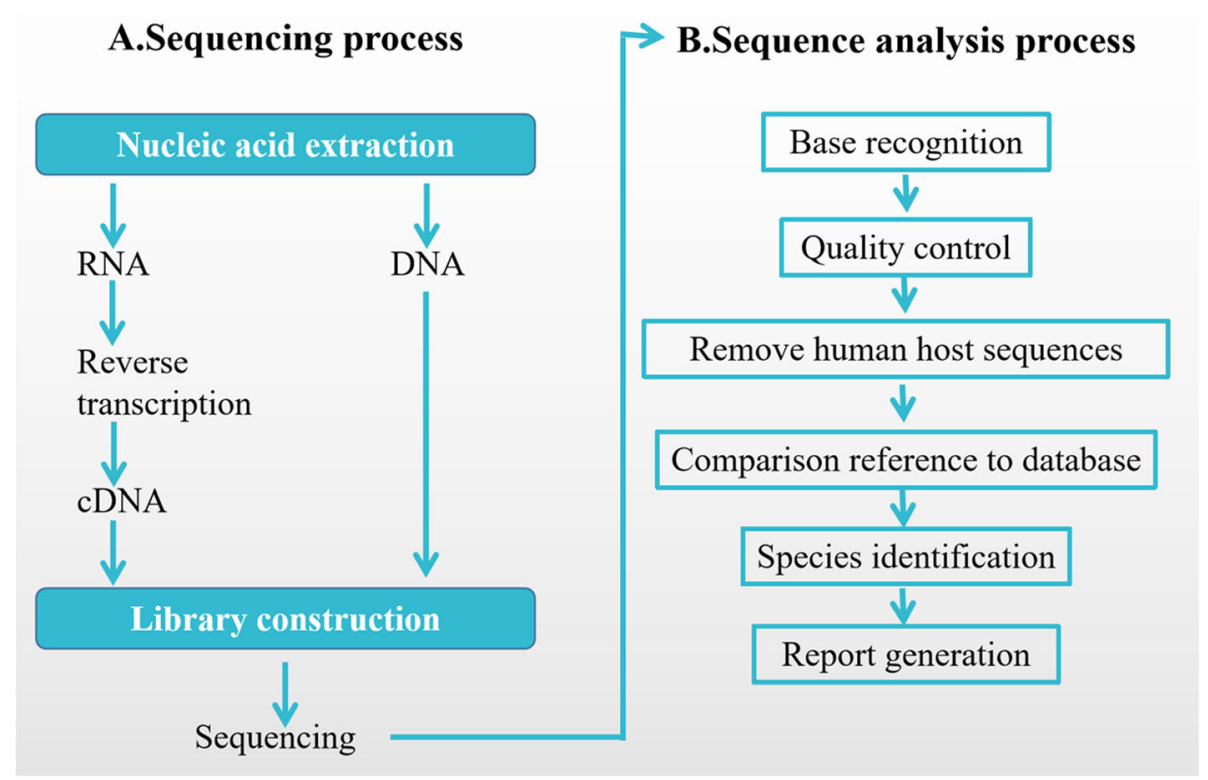

Fig. 2 Flow diagram of Metagenomic Next-generation Sequencing and Analysis 
Sputum samples were liquefied by using $0.1 \%$ dithiothreitol (DTT) for $30 \mathrm{~min}$ at room temperature after inactivation [5]. After that, DNA were extracted by TIANamp Micro DNA Kit (DP316, Tiangen Biotech) according to the manufacturer's recommendation. DNA libraries were constructed in steps of DNA fragmentation by enzyme digestion, DNA supplementation terminal, dA tail and sequencing common connector connection. The constructed DNA library was used to obtain the sequence data of DNA fragments by gene sequencing instrument, and the results were analyzed by biological information software. Each trial included internal, negative and positive controls. Internal parameters is a specific molecular tag that is placed in the sample before nucleic acid extraction to track the entire process and to control the quality of DNA. The detection results of negative control products should be no pathogens detected. If there are relevant pathogens detected, it indicates that there may be DNA pollution sources in the environment. Positive contained specific microbic DNA.

\section{Bioinformatic analysis (Fig. 2b)}

Quality control A. Sequencing subtracted of human host sequences need to be above $90 \%$; B. Reads of microbial detection sequences need to be longer than $50 \mathrm{bp}$ and the effective sequencing data volume should not be less than $20 \mathrm{M}$ without removing the human genome component.

Data filtering In order to obtain high quality sequence data, the qualified data was further filtered by bioinformatics analysis to remove low quality sequences. FASTQ format was used for analysis. The initial pretreatment steps include low quality read filtering, low- complexity read filtering and adapter trimming. Host subtraction was performed by mapping to host genome and/or transcriptome. The remaining unmapped reads are aligned directly with large reference databases, such as the National Center for Biotechnology Information (NCBI) GenBank database.

Sequences alignment The filtered sequences were compared with the reference sequences in the pathogen database, which covers bacteria, fungi, viruses, protozoa and other pathogenic microorganisms. According to the final results of pathogen comparison, all parameters of detected pathogens were calculated, including sequence number, relative abundance, genome coverage and depth, etc.

Report generation The species listed in the report were all the microorganisms detected in this test. They were classified by bacteria, viruses, fungi, parasites, mycoplasma, chlamydia and rickettsia. They were ranked from high to low according to their reads and the relative content of the former is higher. When the report goes to the clinic, whether the suspected pathogen detected is related to infection from the clinical dimension was judged, and the final diagnosis was determine by combining the detection parameters.

\section{Determination of cytokines}

Detection of TNF-a, IL-2, IL-4, IL-6, IL-8, IL-10, IL-17A and INF-r in serum was by solid phase, sandwich and chemiluminescence using the IMMULITE/IMMULIE 1000 analyzer. The analyzer and chemiluminescence kit were both from SIEMENS, Germany. The processed specimens were sent to the analyzer for testing according to the manufacturer's instructions, and the corresponding cytokine concentrations were recorded.

\section{Cell classification and count detection}

Cells were classified using the automatic flow cytometer (Thermo Fisher SCIENTIFIC, American) and divided into total white blood cells, neutrophil count, CD4+ T cell count, CD8+ T cell count, B cells, and NK, T cell count.

\section{Statistical analysis}

Comparative analysis was conducted by Pearson $\chi^{2}$ test and $\mathrm{t}$ test. Data analysis was performed by using SPSS 22.0 software. $P$ values $<0.05$ were considered significant, and all tests were 2-tailed. Logistic regression analysis explored the risk factors associated with positive detection of mNGS.

\section{Results}

\section{Sample and patient characteristics}

Demographic features of the patients were provided in Table 1. 87 males and 22 females participated in our study, whose average age was 61 years old, average length of stay was 17.5 days and the case fatality rate were $11.9 \%$. Most $(37 / 109,33.9 \%)$ of our samples were from blood, 36 of 109 (33.0\%) were from BALF, 12 of $109(11.0 \%)$ were from tissue and $9(8.3 \%)$ of 109 were from sputum, followed by pleural fluid $(7,6.4 \%)$, CSF (4, $3.7 \%)$, pus $(2,1.8 \%)$, bone marrow $(1,0.9 \%)$ and nasal swab (1, 0.9\%) (Fig. 3a). In the study cohort, 92 (84.4\%) patients diagnosed with confirmed pathogens by clinicians were assigned to ID group. The remaining specimens were subdivided into the NID $(16 / 109,14.7 \%)$ and unknown $(1 / 109,0.9 \%)$ groups (Fig. $3 \mathrm{~b})$. There were no statistical differences between ID group and NID group in age, gender, length of stay and case fatality rate $(p>$ 0.05 in all). Most patients were diagnosed with respiratory system infections $(73 / 109,67.0 \%)$, followed by bloodstream infections (10/109, 9.17\%), pleural effusion 
Table 1 Demographic characteristic of samples

\begin{tabular}{llllll}
\hline & Total & ID & NID & Unknown & $P$ value between ID \& NID \\
\hline Samples amount, $\mathrm{n}(\%)$ & $109(100 \%)$ & $92(84.40)$ & $16(14.68)$ & $1(0.92)$ & $/$ \\
Age, average years (range) & $61.02(25-95)$ & $60.26(25-95)$ & $66(40-90)$ & $61(/)$ & 0.43 \\
Gender,male, $\mathrm{n}(\%)$ & $87(79.82)$ & $74(80.43)$ & $12(75.00)$ & $1(100 \%)$ & 0.62 \\
Length of stay, average days (range) & $17.53(1-70)$ & $16.88(1-70)$ & $20.87(6-61)$ & $22(/)$ & 0.31 \\
case fatality rate, \% & 11.93 & 13.04 & 6.25 & 0 & 0.39 \\
\hline
\end{tabular}

Abbreviations: ID infectious disease, NID noninfectious disease

$(6 / 109,5.50 \%)$ and central nervous system infections $(6 /$ 109, 5.50\%) as shown in Fig. 3c.

Diagnostic performance comparison of $\mathrm{mNGS}$ and culture Comparison of diagnostic performance for differentiating ID from NID

The cases of mNGS and culture tests in this study were illustrated in Fig. 4a. In the chi-square test of positive rate, there were statistical differences between mNGS and culture of all and of ID group, but no differences in
NID and unknown group for the limited amounts. 105 samples were included for further study to compare the diagnostic efficiency for differentiating ID from NID. The positive predictive values and negative predictive values of diagnosing infectious disease by mNGS were 92.3 and $27.5 \%$, respectively. The positive likelihood ratio and negative likelihood ratio being 2.16 and 0.47 . The results showed that $\mathrm{mNGS}$ increased the sensitivity rate (positive number in ID/ID number) by approximately $44 \%$ compared with that of culture $(67.4 \%$ vs

\section{A Distribution of Sample Types}

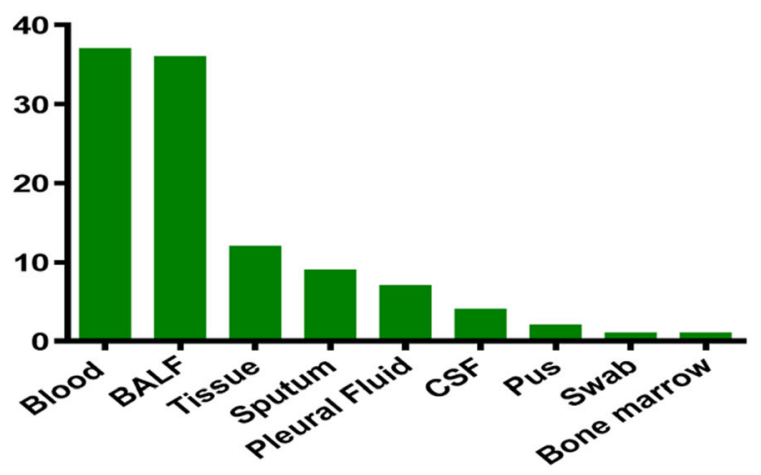

B

C
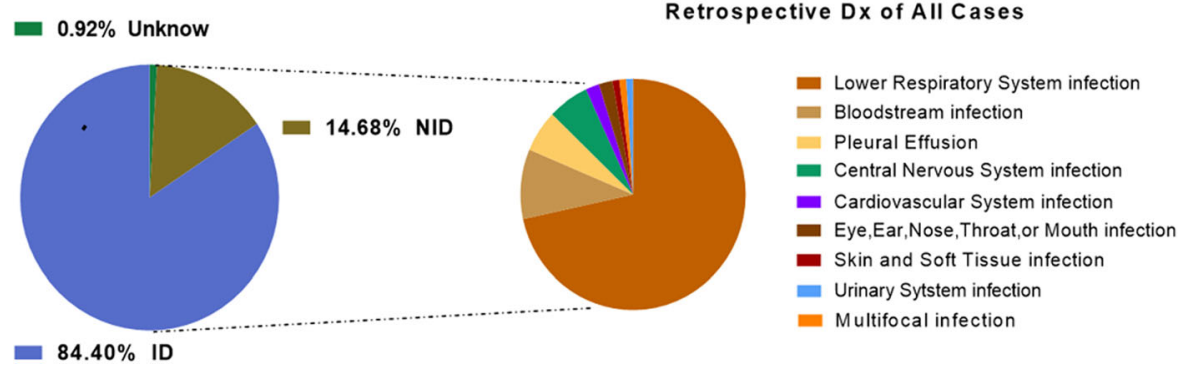

Fig. 3 Patients composition and samples types. a. In samples of this study, 33.9\% were from blood which was the most, 33.0\% from BALF, 11.0\% from tissue and the others were from sputum (8.3\%), pleural fluid (6.4\%), CSF (3.7\%), pus (1.8\%), bone marrow (0.9\%) and nasal swab (0.9\%). b. Patients were subdivided into ID (92/109, 84.4\%), NID (16/109, 14.7\%) and unknown (1/109, 0.9\%) groups according to their diagnosis by conventional technique. $\mathbf{c}$. Infection sites of patients in ID group. Most were respiratory system infections (73/109, 67.0\%) and followed by bloodstream infections (10/109, 9.17\%), pleural effusion (6/109,5.50\%), central nervous system infections (6/109, 5.50\%), cardiovascular system infection (2/109,1.83\%), eye, ear, nose, throat, or mouth infection (2/109,1.83\%), skin and soft tissue infection (1/109, 0.92\%), multifocal infection (1/ 109, 0.92\%), urinary system infection (1/109, 0.92\%). Abbreviations: CSF, cerebrospinal fluid; BALF, bronchoalveolar lavage fluid 


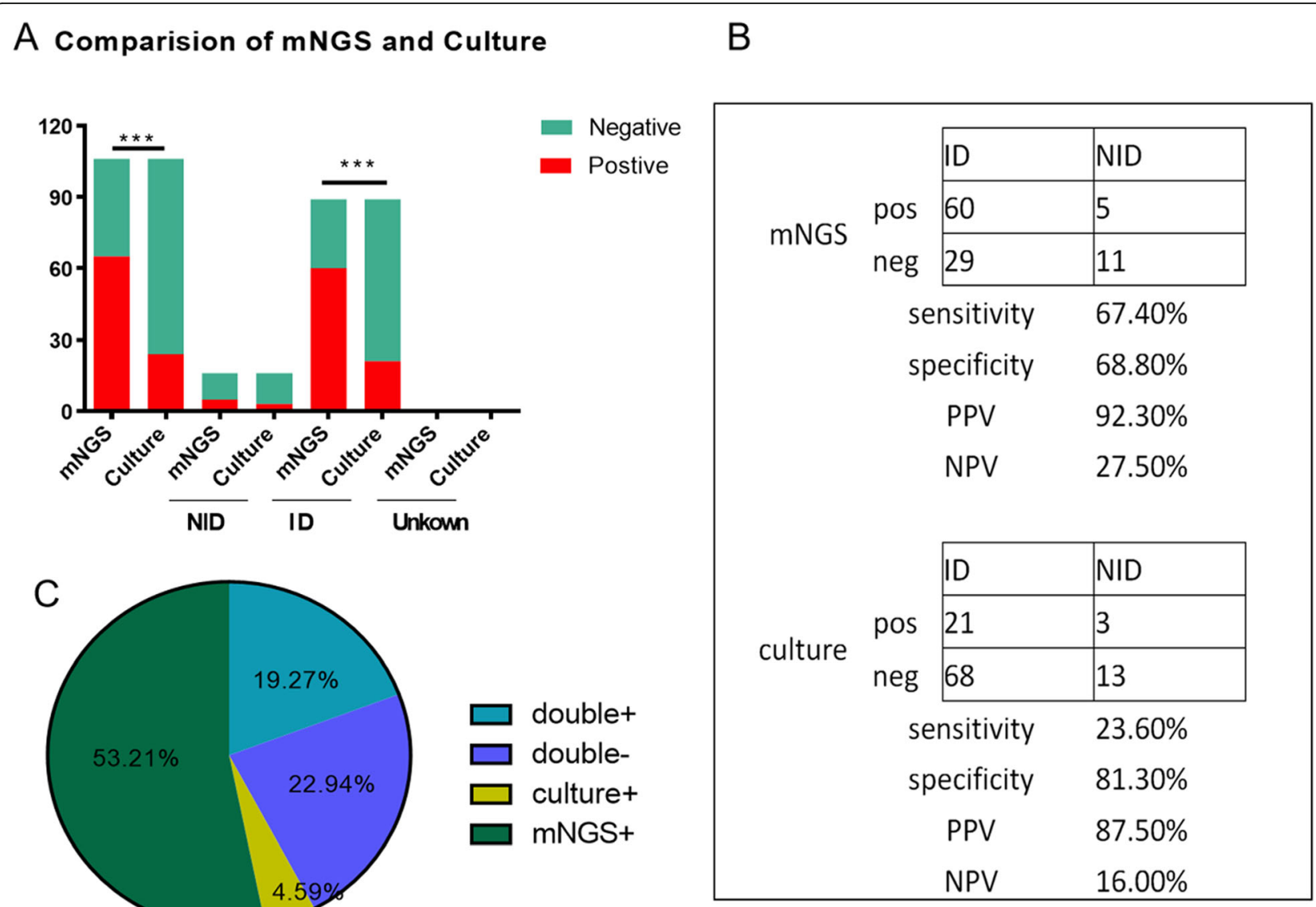

Fig. 4 Diagnostic Performance Comparison of mNGS and Culture. a. Positive and negative cases in all, ID, NID and unknown group of mNGS and the culture, respectively. There were statistical differences between mNGS and culture of all $(P<0.01)$ and of ID group $(P<0.01)$, but no differences in NID and unknown group for the limited amounts $(P>0.05)$. b. Contingency tables showed the sensitivity and specificity of mNGS were 67.4 and $68.8 \%$, while those of culture were 23.6 and $81.3 \%$. mNGS increased the sensitivity in comparison with that of culture $(P<0.001)$ while there were no differences in specificity between them $(P=0.41)$. c. Pie chart demonstrated the positivity distribution of mNGS and culture for all samples from 3 groups. 53.21\% were positive by mNGS, $4.59 \%$ by culture, $19.27 \%$ by both and $22.94 \%$ were both negative. Abbreviations: NPV, negative predictive values; PPV, positive predictive values

23.6\%; $P<0.001)$ and decreased the specificity rate (negative number in NID /NID number) by $12.5 \%$ compared with that of culture $(68.8 \%$ vs $81.3 \%$; $P=$ 0.41) (Fig. 4b).

\section{Concordance between mNGS and culture for pathogen detection}

In this study, mNGS and culture were both positive in 21 of $109(19.3 \%)$ cases and were both negative in 25 of $109(22.9 \%)$ cases. There were 58 cases $(53.2 \%)$ were positive by mNGS only and $5(4.6 \%)$ were positive only by culture. The 2 results in double-positive cases were completely matched (overlapped of all pathogens) in 3 of 21 and totally mismatched (overlapped of no pathogen) in 3 of 21 (Fig. 4c). The remaining 15 cases were found to at least one but not all overlapped of pathogens in polymicrobial results, which defined as "partly matched".
"False positives" and "false negatives" of mNGS

In the ID group, three culturable pathogens were missed by mNGS. Among the three "mNGS false-negative" samples, there were 2 culture results paradoxical with clinical diagnosis, the other 1 was completely unidentified by mNGS. At the same time, the possible reasons for the 7 cases of "mNGS false-positive" in the NID group included potential concomitant infection with NIDs (3/7), overinterpretation (3/7) and unknown (1/7) (Table 2).

\section{Comparison of mNGS and culture testing by pathogens and samples}

Comparison analysis at the pathogen-type level

Klebsiella (10/69) was the most commonly detected pathogen among the 69 microbes isolated in mNGS and culture testing, followed by bacteria without MTB/NTM (9/69), Aspergillus (6/69), Pseudomonas (6/69) and EBV (6/69) (Fig. 5a). The percentage of mNGS-positive 
Table 2 "False Positives" and "False Negatives" of mNGS

\begin{tabular}{|c|c|c|c|c|}
\hline \multicolumn{5}{|c|}{ Pathogens Detected Only by mNGS in the NID Group } \\
\hline $\begin{array}{l}\text { Sample } \\
\text { No. }\end{array}$ & $\begin{array}{l}\text { Specimen } \\
\text { source }\end{array}$ & Diagnosis & mNGS result & Possible explanation \\
\hline 2 & BALF & Hematencephalon & Acinetobacter baumannii, Klebsiella, Enterococcu & Unknown \\
\hline 33 & Blood & Lymphoma & Pseudomonas, CMV & $\begin{array}{l}\text { Potential cause of } \\
\text { lymphoma }\end{array}$ \\
\hline 62 & Blood & Aplastic anemia & Acinetobacter baumannii, Enterococcu & Overinterpretation \\
\hline 67 & Blood & myelofibrosis & Phycomyces blakesleeanus & Overinterpretation \\
\hline 74 & Pleural Fluid & Pleural effusion & $\begin{array}{l}\text { Fusobacterium nucleatum, Streptococcus constellatus, } \\
\text { Porphyromonas gingivalis }\end{array}$ & $\begin{array}{l}\text { Potential cause of } \\
\text { inflammation }\end{array}$ \\
\hline 86 & Blood & Ulcerative Colitis & Porphyromonas gingivalis, HSV & $\begin{array}{l}\text { Potential cause of } \\
\text { inflammation }\end{array}$ \\
\hline 88 & Blood & Lung cancer & Saccharomyces cerevisiae & Overinterpretation \\
\hline \multicolumn{5}{|c|}{ Culturable Pathogens Missed by mNGS in the ID Group } \\
\hline \multicolumn{2}{|l|}{ Microbe } & Count & \multicolumn{2}{|l|}{ Possible explanation } \\
\hline \multicolumn{2}{|l|}{ MTB } & 2 & \multicolumn{2}{|l|}{ Positive Not Detected } \\
\hline \multicolumn{2}{|c|}{ Pseudomonas } & 1 & \multicolumn{2}{|l|}{ Microbes "Weak" } \\
\hline
\end{tabular}

Abbreviations: $m N G S$ metagenomic next-generation sequencing, ID infectious disease, NID noninfectious disease, $C M V$ metagenomic next-generation sequencing, HSV herpes simplex virus, MTB Mycobacterium tuberculosis

samples observed to have a higher yield rate than that of culture, but the differences were not significant $(P>0.05)$ in terms of Klebsiella, bacteria without MTB/NTM, EBV, CMV due to the small sample size. In Acinetobacter baumannii $(n=2)$ and MTB $(n=3)$, the number of mNGS-positive samples was equally with that of culture-positive samples. While only mNGS indicated positive results in NTM $(n=4)$, Anaerobes $(n=4)$, Saccharomyces cerevisiae $(n=2)$, Proteus $(n=1)$, Pneumocystis carinii $(n=$ $2)$, Abiotrophia $(n=1)$, Nocardia $(n=3)$, Staphylococcus aureus $(n=2)$, Enterococcu $(n=2)$ and Escherichia coli $(n=1)$.

\section{Comparison analysis at the sample-type level}

In the types of BALF, tissue, blood and sputum samples, mNGS detection had significantly higher sensitivity than the culture method $(P=0.002$ for BALF, $P=0.025$ for tissue, $P<$ 0.001 for blood, $P=0.018$ for sputum), and the overall sensitivity of mNGS in the sample types was significantly different $(P=0.03)$. In the types of pleural fluid, CSF, pus, bone marrow and nasal swab, there were no significant differences in sensitivity between two methods $(P>0.05)$. In addition, in the culture method, the positive rate in BALF was higher than that in the whole blood $(P=0.019)$, and there was no difference in the overall sensitivity of the culture method in the sample type, as shown in Fig. 5b.

Comparison of infection indexes in positive and negative group by mNGS in ID

Classification and counting of leukocyte and lymphocyte in positive and negative group by $m$ NGS

In this study, complete blood count, CRP and PCT tests were examined on the day of examination of pathogenic microorganisms to determine the differences in the total number of white blood cells, lymphocytes and neutrophils between the positive group and the negative group by mNGS. The results showed (Table 3) that there were no statistically differences in leukocyte and lymphocyte between positive and negative groups by mNGS $(P>0.05)$.

\section{Comparison of cytokine concentrations in positive and negative group by $\mathrm{mNGS}$}

In order to explore the correlation between the status of immune function in patients and the positive results of pathogen examination, this study detected and analyzed the peripheral blood (TNF-a, IL-2, IL-4, IL-6, IL-8, IL-10, IL-17A and INF-r) in infected patients. The results indicated that the peripheral blood concentrations of IL-10 in the positive group was higher than that in the negative group, and the differences were statistically significant $(P=0.044)$, while other cytokine showed no difference between groups as shown in Table 3.

\section{Analysis of correlative factors for positive result of pathogen extraction by $\mathrm{mNGS}$}

In order to further explore the related risk factors of positive mNGS test in infected patients, this study used Logistic multivariate regression analysis to analyze the patients' information and whether the pathogen was detected in the patients. After the confounding factors were removed, the variables that were significant for detection was age $(P=0.037, \mathrm{OR}$ : 1.076, 95\% CI:1.005-1.152), which promoted the detection of pathogens (Table 4). 


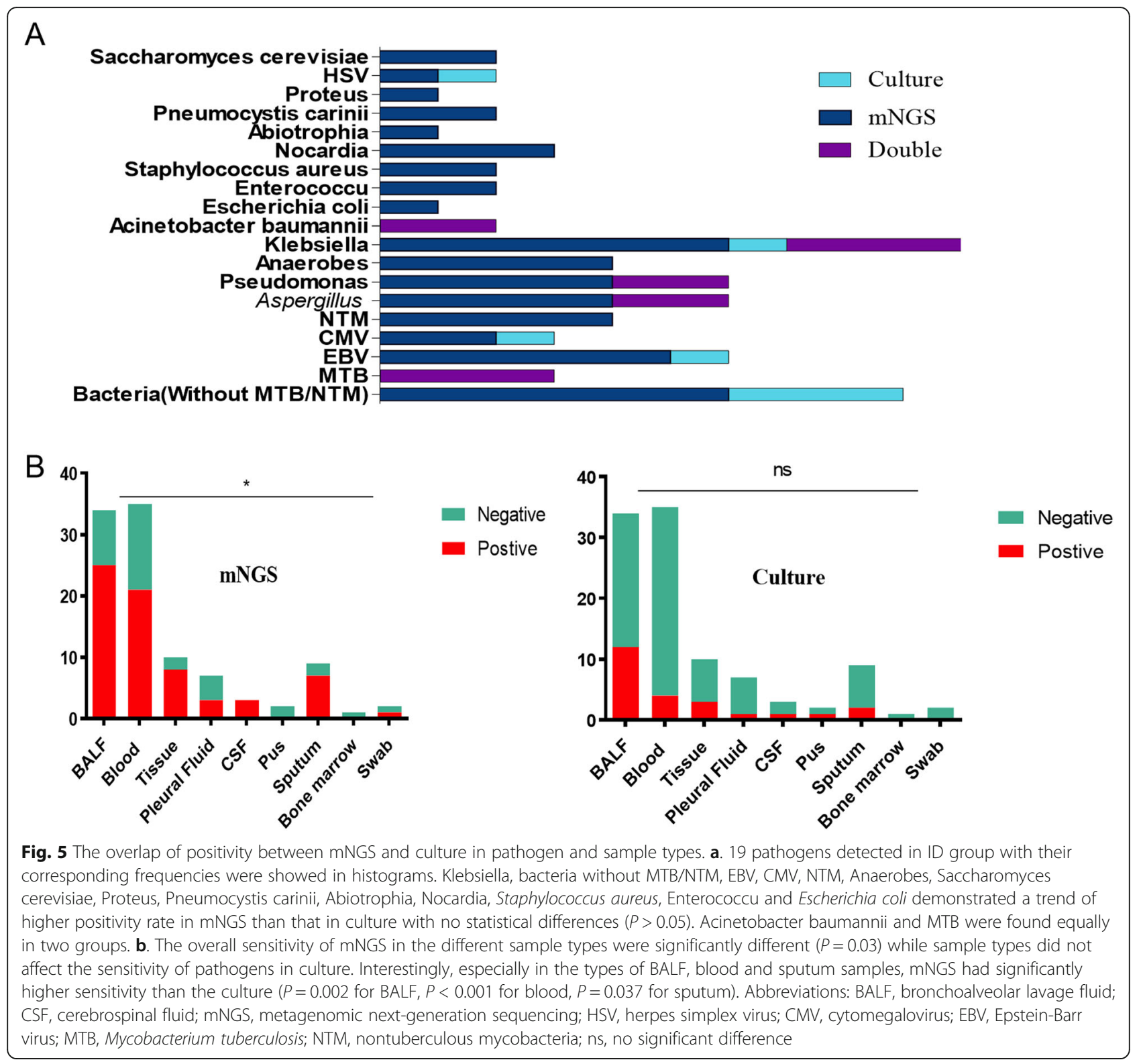

Potential implications of clinical mNGS test Potential inappropriate antibiotic usage for patients with virus isolates

There were 4 viruses identified by mNGS from 23 patients in this study, the majority of the identified viruses were herpes simplex virus $(n=15)$, followed by EpsteinBarr virus/ herpes simplex virus $(n=5)$, Epstein-Barr virus $(n=1)$, Hepatitis A virus $(n=1)$ and torque teno virus $(n=1)$. Nearly $50 \%$ of patients were diagnosed with a hospital-acquired infection (12/23) and 17 of 23 patients were given broad-spectrum antibiotics based on symptoms, imaging. 10 of 23 patients were suspected of inappropriate antibiotic usage, which means after broadspectrum antibiotic treatment, patients' symptoms did not improve or even worsened and after identifying the real pathogen through mNGS and adjusting the antibiotic use based on that, patients' condition improved. 7 of 23 were considered immunocompromised hosts characterized by deficiency of the immune system or immune response caused by infectious factors, mycotoxins, drugs and nutritional deficiencies. (Table 5).

The influence of positive by mNGS on the hospital days and survival of patients

As Table 6 showed, there were 67 samples in positive group with 57 males and 26 in negative group with 20 males. There was no significant difference in mean age between the two groups $(59.70$ yrs. vs 60.50 yrs., $P=$ 0.84). Positive group had a longer hospital day (HOD, 176.63 days vs 150.96 days, $P=0.047$ ) and a higher 28- 
Table 3 The counts of WBC, Cytokines and lymphocytes in positive and negative groups by mNGS

\begin{tabular}{|c|c|c|c|}
\hline & Positive & Negative & $\mathbf{P}$ \\
\hline \multicolumn{4}{|c|}{ Cytokines pg/ml } \\
\hline $\mathrm{IL}-2$ & $100.35 \pm 68.21$ & $1.31 \pm 0.94$ & 0.511 \\
\hline $\mathrm{IL}-4$ & $2.74+0.41$ & $1.52+0.94$ & 0.206 \\
\hline IL-6 & $70.8 \pm 18.27$ & $68.96 \pm 33.18$ & 0.964 \\
\hline TNF-a & $2.48 \pm 0.42$ & $2.26 \pm 1.32$ & 0.842 \\
\hline IL-17a & $13.77 \pm 2.35$ & $10.45 \pm 8.01$ & 0.592 \\
\hline IL-8 & $1154 \pm 0$ & - & - \\
\hline$\| \mathrm{L}-10$ & $26.14+7.75$ & $8.29 \pm 3.33$ & 0.044 \\
\hline $\mathrm{IFN}-\gamma$ & $8.91+1.89$ & $13.59 \pm 6.92$ & 0.361 \\
\hline \multicolumn{4}{|c|}{ Cellular Immunity \% } \\
\hline CD4/CD8 & $1.42 \pm 0.23$ & $2.06 \pm 0.44$ & 0.185 \\
\hline Th cell & $35 \pm 3.36$ & $43.83 \pm 5.75$ & 0.201 \\
\hline Ts cell & $68.06 \pm 3.07$ & $66.67 \pm 3.64$ & 0.18 \\
\hline NK cell & $15.71 \pm 2.17$ & $15.5 \pm 1.89$ & 0.958 \\
\hline B cell & $13.53 \pm 2.94$ & $12.83+3.73$ & 0.899 \\
\hline T cell & $68.06 \pm 3.07$ & $66.67 \pm 3.64$ & 0.958 \\
\hline WBC ${ }^{\times} 109$ & $8.32 \pm 0.52$ & $7.36 \pm 0.48$ & 0.283 \\
\hline $\mathrm{Neu}^{\times} 109$ & $6.99 \pm 0.58$ & $5.38 \pm 0.48$ & 0.109 \\
\hline $\mathrm{PCT} \mathrm{ng} / \mathrm{ml}$ & $0.34 \pm 0.17$ & $3.42 \pm 3.32$ & 0.112 \\
\hline CRP mg/l & $87.63 \pm 8.32$ & $63.61 \pm 13.47$ & 0.129 \\
\hline
\end{tabular}

Abbreviations: $m N G S$ metagenomic next-generation sequencing, $W B C$ white blood cells, IL- interleukin-, IFN- $\gamma$ Interferon- $\gamma$, TNF- $a$ Tumor Necrosis Factor-a, CD4 Cluster of Differentiation 4 receptors, CD8 Cluster of Differentiation 8 receptors, Th helper T cell, Ts suppressor T cell, NK natural killer cell, Neu neutrophil, $P C T$ procalcitonin, CRP C-reactive protein

day mortality $(9.0 \%$ vs $0 \%, P=0.049)$ than those of negative group, but there were no statistical differences in 14-day mortality $(4.5 \%$ vs $0 \%, P=0.278)$ and 90 -day mortality $(13.4 \%$ vs $3.9 \%, P=0.180)$ between groups. The average survival time of two groups were 176.64 days and 150.96 days, respectively, but $P$ value for $\mathrm{t}$ test between groups was 0.425 , no statistical differences. The survival curves of the two groups were shown in Fig. 6 . At the meantime, we analyzed the relationship between pathogens read number and HOD, 14-day-mortality, 28day-mortality and 90-day-mortality, which showed that the higher pathogens read number, the higher 90-daymortality and the longer HOD (Table 7).

\section{Discussion}

The traditional clinical model for diagnosing infectious diseases is for doctors to make a differential diagnosis and then conduct a series of tests to try to identify the pathogen [9-12]. Traditional diagnostic techniques of microbiology laboratory ranges from smear microscopy, microorganisms' culture, antigen antibody detection and PCR mainly. Whereas most traditional methods were often time-consuming and has a lower positive rate than
Table 4 The analysis of the relevant factors of pathogens DNA positive in patients

\begin{tabular}{lllllll}
\hline Values & $\mathbf{B}$ & $\mathbf{S E}(\mathbf{B})$ & $\mathbf{W a l d} \mathbf{X}^{\mathbf{2}}$ & $\mathbf{P}$ & $\mathbf{O R}$ & $\mathbf{9 5 \%} \mathbf{C l}$ \\
\hline Age & 0.073 & 0.035 & 4.367 & 0.037 & 1.076 & $1.005-1.152$ \\
Sex & -0.545 & 1.157 & 0.222 & 0.637 & 0.58 & $0.06-5.601$ \\
Read Number & -2.371 & 0.599 & 15.677 & 0.000 & 0.093 & $0.029-0.302$ \\
HOD & -0.028 & 0.061 & 0.216 & 0.642 & 0.972 & $0.863-1.095$ \\
Survival Time & -0.007 & 0.005 & 1.888 & 0.169 & 0.993 & $0.983-1.003$
\end{tabular}

Cytokines pg/ml

\begin{tabular}{lllllll} 
IL-2 & 0.171 & 1.115 & 0.023 & 0.878 & 1.186 & $0.133-10.553$ \\
IL-4 & -1.299 & 0.893 & 2.116 & 0.146 & 0.273 & $0.047-1.57$ \\
IL-6 & -0.005 & 0.019 & 0.077 & 0.781 & 0.995 & $0.957-1.033$ \\
TNF-a & -0.374 & 0.373 & 1.003 & 0.316 & 0.688 & $0.331-1.430$ \\
IL-17a & 0.202 & 0.137 & 2.165 & 0.141 & 1.223 & $0.935-1.6$ \\
IL-10 & -2.64 & 0.206 & 1.639 & 0.201 & 0.768 & $0.513-1.151$ \\
IFN-y & 0.09 & 0.071 & 1.606 & 0.205 & 1.095 & $0.952-1.259$ \\
Cellular Immunity \% & & & & & \\
CD4/CD8 & -0.488 & 0.965 & 0.256 & 0.613 & 0.614 & $0.093-4.067$ \\
Th cell & 0.318 & 0.296 & 1.151 & 0.283 & 1.374 & $0.769-2.454$ \\
Ts cell & 0.244 & 0.317 & 0.589 & 0.443 & 1.276 & $0.685-2.377$ \\
NK cell & -0.223 & 0.211 & 1.121 & 0.29 & 0.800 & $0.529-1.209$ \\
B cell & $-0.26-$ & 0.245 & 1.172 & 0.279 & 0.767 & $0.227-1.475$ \\
T cell & 0.5485 & 0.478 & 1.315 & 0.252 & 0.578 & $0.475-1.239$ \\
WBC ${ }^{\times}$109 & -0.123 & 1.228 & 0.01 & 0.92 & 0.884 & $0.08-9.819$ \\
Neu ${ }^{\times 109}$ & 0.141 & 1.39 & 0.01 & 0.919 & 1.151 & $0.076-17.535$ \\
PCT ng/ml & -0.681 & 1.514 & 0.202 & 0.653 & 0.506 & $0.026-9.844$ \\
CRP mg/l & -0.004 & 0.015 & 0.073 & 0.788 & 0.996 & $0.968-1.025$ \\
\hline Ab & & &
\end{tabular}

Abbreviations: $H O D$ hospital day, WBC white blood cells, IL-interleukin-, IFN- $\gamma$ Interferon- $\gamma$, TNF- $a$ Tumor Necrosis Factor- $a$, CD4 Cluster of Differentiation 4 receptors, $C D 8$ Cluster of Differentiation 8 receptors, Th helper T cell, $T s$ suppressor T cell, NK natural killer cell, Neu neutrophil, PCT procalcitonin, CRP C-reactive protein

mNGS [2-4]. Although molecular diagnostic assays are a quick way to diagnose the most common infections, almost all conventional microbial trials in use today only target a limited number of pathogens at a time or require successful culture of microorganisms from clinical samples [13]. While mNGS analyze the entire microbiome in patients' samples [8] so it has been used to discover novel viral pathogens and diagnose viral infections in people widely [14-16]. Therefore, we explored the application and differences between traditional culture method and mNGS in clinical infectious diseases in adults. BALF, blood, sputum, tissue, CSF, pleural fluid, pus, bone marrow or nasal swab from 109 patients suspected of infection were collected and specimens were subjected to regular clinical microbiological assay and mNGS testing in a pairwise manner in our study. We then systematically compared the clinical features and test results of mNGS and traditional culture. 
Table 5 Clinical Characteristics of Patients with Virus Isolates $(n=23)$

\begin{tabular}{|c|c|c|c|c|c|c|c|c|c|c|}
\hline \multirow[t]{2}{*}{ Type of Virus } & \multicolumn{2}{|l|}{ HAI } & \multicolumn{2}{|c|}{$\begin{array}{l}\text { Immunosuppressed } \\
\text { Patients }\end{array}$} & \multicolumn{2}{|c|}{$\begin{array}{l}\text { Broad-spectrum } \\
\text { Antibioticsa }\end{array}$} & \multicolumn{2}{|c|}{ Suspected Inappropriate Antibiotic Usage } & \multicolumn{2}{|c|}{ Treatment Responsive } \\
\hline & Yes & No & Yes & No & Yes & No & Yes & No & Yes & No \\
\hline $\operatorname{HSV}(n=15)$ & 8 & 7 & 7 & 8 & 10 & 5 & 5 & 10 & 8 & 7 \\
\hline $\operatorname{HAV}(n=1)$ & 1 & 0 & 0 & 1 & 1 & 0 & 1 & 0 & 1 & 0 \\
\hline HSV/EBV $(n=5)$ & 3 & 2 & 0 & 5 & 4 & 1 & 2 & 3 & 3 & 2 \\
\hline $\operatorname{TTV}(n=1)$ & 0 & 1 & 0 & 1 & 1 & 0 & 1 & 0 & 0 & 1 \\
\hline EBV $(n=1)$ & 0 & 1 & 0 & 1 & 1 & 0 & 1 & 0 & 0 & 1 \\
\hline Total $(N=23)$ & 12 & 11 & 7 & 16 & 17 & 6 & 10 & 13 & 12 & 11 \\
\hline
\end{tabular}

Abbreviations: EBV Epstein-Barr virus, $H A I$ hospital-acquired infection, $H S V$ herpes simplex virus, $H A V$ herpes simplex virus, $T T V$ torqueteno virus

The results suggested that there were no significant differences in age, gender, length of stay and fatality rate between two groups and mNGS had an advantage in sensitivity rate compared with traditional culture method. A team of researchers also found that mNGS detected potential pathogenic bacteria, which had advantages in speed and sensitivity compared with culture and pathology [17], Miao's team [5] showed that mNGS had a sensitivity of $50.7 \%$ for the diagnosis of infectious diseases, higher than traditional culture (50.7\% vs $35.2 \%)$. In particular, the diagnosis of MTB, virus, anaerobic bacteria, nocardia and fungi has obvious advantages. The results were similar to our results, which showed that the sensitivity of mNGS was $67.4 \%$, significantly higher than that of culture method (23.6\%). High sensitivity of mNGS may because pathogen DNA has a long survival time in plasma, the use of antibiotics has a small impact on mNGS results, while traditional cultures are greatly affected by the use of antibiotics. Because of the small sample size, mNGS showed no statistical difference compared with culture method in pathogen types although there was a trend of superiority in Klebsiella, bacteria without MTB/NTM, EBV, CMV, NTM,

Table 6 The basic demographic and clinical characteristics of initial and outcome patient variables

\begin{tabular}{llll}
\hline & Positive & Negative & P \\
\hline Sex & & & \\
Female & 10 & 6 & 0.355 \\
Male & 57 & 20 & \\
Age & $59.70 \pm 2.16$ & $60.50 \pm 3.06$ & 0.84 \\
HOD & $176.63 \pm 17.70$ & $150.96 \pm 103.14$ & 0.047 \\
14 days of death & $4.5 \%$ & 0 & 0.278 \\
28 days of death & $9.0 \%$ & 0 & 0.049 \\
90 days of death & $13.4 \%$ & $3.9 \%$ & 0.180 \\
Read Number & $5295.62 \pm 2507.26$ & $16.67 \pm 4.79$ & 0.039 \\
Survival time & $176.64 \pm 17.70$ & $150.96 \pm 21.05$ & 0.425 \\
\hline
\end{tabular}

Abbreviation: $H O D$ hospital day
Anaerobes, Saccharomyces cerevisiae, Proteus, Pneumocystis carinii, Abiotrophia, Nocardia, Staphylococcus aureus, Enterococcus and Escherichia coli. However, mNGS detection had a significantly higher sensitivity than the culture method in BALF $(P=0.002)$, tissue $(P=0.025)$, blood $(P<0.001)$ and sputum $(P=0.018)$ samples.

Based on the advantages shown by mNGS, we then investigated the influence of positive mNGS detection results on the severity and prognosis of patients with infection. By comparing the classification and counting of leukocyte, lymphocyte and cytokine concentrations in positive and negative groups, we found that IL-10 concentration in peripheral blood in the positive group was higher than that in the negative group and there were no statistically differences in other cytokine concentrations, leukocyte and lymphocyte. According to the results of correlative factors analysis for positive test of mNGS, patients' age may promote the detection of pathogens. In the survival analysis, positive group had a higher 28 -day mortality $(9.0 \%$ vs $0 \%, P=0.049)$ than that

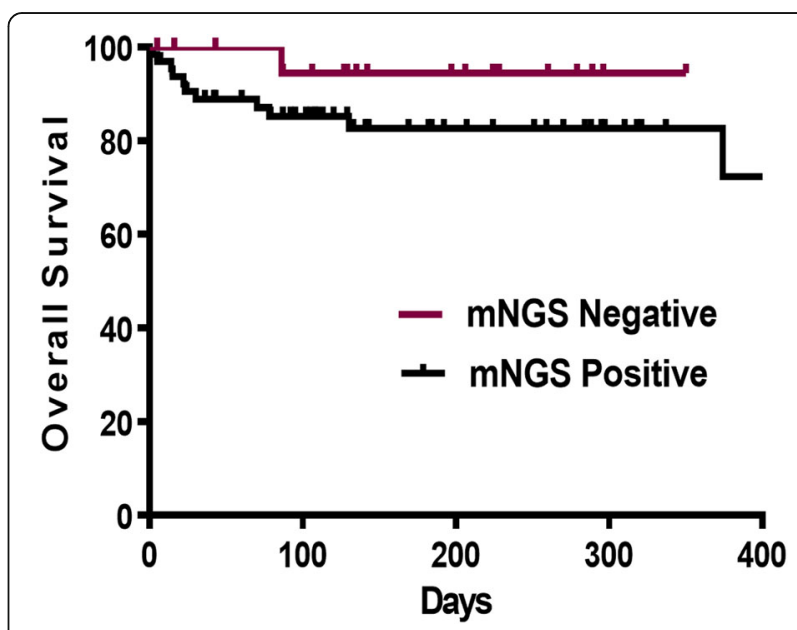

Fig. 6 The survival curves of positive and negative group of mNGS in ID. The survival curves suggested that the overall survival rate declined faster in the positive group, however, there was no statistically differences between the two groups 
Table 7 The analysis between the pathogens read number and HOD,14, 28 and 90-day-mortality

\begin{tabular}{|c|c|c|c|c|c|c|c|}
\hline \multicolumn{8}{|c|}{ Read Number } \\
\hline & 0 & $1-9$ & $10-99$ & 100-999 & $1000-$ & $\mathrm{F}$ & $P$ \\
\hline No & 20 & 15 & 20 & 24 & 14 & & \\
\hline $\mathrm{HOD}$ & $14.84 \pm 8.58$ & $13.07 \pm 5.18$ & $15.80 \pm 9.12$ & $20.70 \pm 16.5$ & $27.92 \pm 24.06$ & 2.685 & 0.037 \\
\hline 14-mortality & 0 & 0 & 0 & 0.04 & 0.14 & 1.898 & 0.118 \\
\hline 28-mortality & 0 & 0.13 & 0.05 & 0.083 & 0.29 & 2.253 & 0.07 \\
\hline 90-mortality & 0.05 & 0.2 & 0.05 & 0.083 & 0.36 & 2.598 & 0.042 \\
\hline Survival Time & $169.74+102.68$ & $138.40+100.27$ & $158.70+125.83$ & $185.45+124.82$ & $194.71+216.79$ & 0.424 & 0.791 \\
\hline
\end{tabular}

Abbreviation: $H O D$ hospital day

of negative group, but there were no statistical differences in average survival time. The pathogens read number by mNGS was positive related to the HOD and 90day-mortality of patients with infectious diseases. All of that indicated older people were more likely to have positive results and positive results of mNGS detection may represent a worse outcome.

Fortunately, mNGS has moved from scientific application to clinical practice and is changing the way disease diagnosed and treated [18-20]. In addition to what we mentioned above, mNGS also has merits in many other aspects. Firstly, mNGS does not need prior clinical information to detect infectious pathogens, and the results can be reported quickly and accurately, greatly shortening the diagnosis time of infectious pathogens. Early and rapid reporting of the results by mNGS provides clinical clues to the next step in diagnosis and treatment, especially avoiding overuse of antibiotics for viral infections $[21,22]$. Rapid results reported by mNGS also can promote timely adjustment of treatment in clinical practice. As our data showed, almost one-half of patients with virus infection were suspected of inappropriate antibiotic usage. Secondly, mNGS was used in some rare infectious pathogens. It detected Naegleria fowleri [23], brucellosis [24], cysticercosis, taenia bocinea [25], gondii [26] in CSF, Hepatic tuberculosis in blood [27] in previous reports. Thirdly, studies have shown that mNGS can be used not only for pathogen identification, but also for microbiome characterization, parallel analyses of human host responses, drug resistance gene and virulence factor detection. All of these led to the rapid development of mNGS in immunodeficiency difficult-to-diagnose cases and immunocompromised patients [13]. Thirdly, antibiotic usage had little influence on mNGS results due to the long survival time of pathogen DNA in plasma, but traditional cultures were affected by antibiotic use [21, 22]. Higher sensitivity of mNGS than culture in this study may because that mNGS is less affected by prior antibiotic usage. However, mNGS still has some limitations at present, such as human background, background bacteria contamination, no uniform standards for detailed experimental procedures [2, 28-31], inability to distinguish infection and colonization, standardization of bioinformatics analysis process, and problem of report interpretation. The results must be interpreted in the context of the clinical situation. It's worth noting that background microbial contamination is a common problem faced by mNGS technology, which can be partially eliminated through negative quality control, but it requires clinical familiarity with common background bacteria and better interpretation results combined with clinical practice [24].

In this study, we systematically compared mNGS and traditional culture method in sensitivity, specificity, pathogen type and sample type. On this basis, we also compared and analyzed the differences between the positive and negative groups of mNGS which was few at present. Patients of positive group found to have a trend of worse prognosis suggested need more attention clinically. Small sample size was the biggest deficiency of our study, so that there were many results indicated a certain trend without reaching statistical significance unfortunately. Therefore, more patients need to be included in the study in the future. Not randomized controled was also the limitation of study. As a retrospective study, this study has some limitations like limited data and data accumulation not controlled by the researcher. Besides, limit generalizability caused by single-center study, lack of a gold standard comparator for diagnostics, lack of antibiotic usage detail and classification bias were also the limitations of this study.

\section{Conclusions}

In summary, mNGS had a higher sensitivity than culture, especially in the types of BALF, blood and sputum samples, and there was a trend of higher sensitivity of Klebsiella, CMV and EBV detection. The worse trend of outcome in patients with positive mNGS results than negative group prompted more clinical attention to patients with positive mNGS results is required. Therefore, based on what we found above and other advantages of mNGS like quick results, less affected by prior antibiotic exposure and so on, we suggest that mNGS should be used more in early pathogen diagnosis in the future. Nonetheless, interpreting data of mNGS will be a challenge for doctors in guiding clinical treatment of infectious diseases. 


\section{Abbreviations}

mNGS: Metagenomic next-generation sequencing; BALF: Bronchoalveolar lavage fluid; CSF: Cerebrospinal fluid; Dx: Diagnosis; ID: Infectious disease; NID: Noninfectious disease; DNA: Deoxyribonucleic acid; EDTA: Ethylene Diamine Tetraacetic Acid; EBV: Epstein-Barr virus; HAl: Hospital-acquired infection; HboV: Human bocavirus; HSV-1: Herpes simplex virus 1; TTV: Torqueteno virus; HAV: Hepatitis A virus; HOD: Hospital day; IL2: Interleukin-2; IL-4: Interleukin-4; IL-6: Interleukin-6; IL-10: Interleukin-10; IL8: Interleukin-10; CD4: Cluster of Differentiation 4 receptors; CD8: Cluster of Differentiation 8 receptors; WBC: White blood cell; Th: Helper T; NK: Natural killer cell; PCT: Procalcitonin; CRP: C-reactive protein; IFN- $\gamma$ : Interferon- $\gamma ;$ IL17a: Interleukin-17a; TNF-a: Tumor Necrosis Factor-a; PPV: Positive predictive value; NPV: Negative predictive value; CMV: Cytomegalovirus; MTB: Mycobacterium tuberculosis; ns: No signicant difference; NTM: Nontuberculous mycobacteria; G + : Gram Positive; G-: Gram Negative

\section{Acknowledgments}

We would like to thank all the patients who donate their biological samples.

\section{Authors' contributions}

Conception and design: H.X.D, S.S.X, X. L, A.H.M. Acquisition, statistical analysis or interpretation of the data: P. L, Y. L, X.F.L, W.W.L. Drafting of the manuscript: C.H.W. All authors reviewed and approved the final version of the manuscript. All authors had read and approved the manuscript.

\section{Funding}

This work was supported by the National Natural Science Foundation of China (No. 81802262), the Fundamental Research Funds for the Central Universities (No. 22120180584), Shanghai Tenth Hospital's Improvement Plan for NSFC (No. 04.03.17.032, 04.01.18.048, SYGZRPY2017014). Changhui Wang is the recipient of the foundations and was responsible for editing manuscript in our study.

\section{Availability of data and materials}

All data generated or analysed during this study are included in this published article. The data that support the findings of this study are available from the corresponding author upon reasonable request.

\section{Ethics approval and consent to participate}

All experiments performed in this study are in accordance with the approved ethical standards by the ethics committee of the 10th People's Hospital affiliated to Tongji University (No. SHSY-IEC-4.1/20-21/01). Formal consent is waived for this type of study.

\section{Consent for publication}

Not Applicable.

\section{Competing interests}

The authors declare that they have no competing interests.

\section{Author details}

'Department of Respiratory Medicine, Shanghai 10th People's Hospital, Tongji University School of Medicine, \#301, Mid Yanchang Rd, Shanghai 200072, China. 2Department of Emergency, Shanghai 10th People's Hospital, Tongji University School of Medicine, Shanghai 200072, China. ${ }^{3}$ Department of Geriatrics, Shanghai 10th People's Hospital, Tongji University School of Medicine, Shanghai 200072, China.

\section{Received: 21 March 2020 Accepted: 27 December 2020} Published online: 13 January 2021

\section{References}

1. Zhou K, Lokate M, Deurenberg RH, Tepper M, Arends JP, Raangs EG, Lo-TenFoe J, Grundmann H, Rossen JW, Friedrich AW. Use of whole-genome sequencing to trace, control and characterize the regional expansion of extended-spectrum beta-lactamase producing ST15 Klebsiella pneumoniae. Sci Rep. 2016:6:20840.

2. Schlaberg R, Chiu CY, Miller S, Procop GW, Weinstock G, Professional Practice $C_{1}$ Committee on Laboratory Practices of the American Society for M, Microbiology Resource Committee of the College of American P.
Validation of metagenomic next-generation sequencing tests for universal pathogen detection. Arch Pathol Lab Med 2017; 141:776-786.

3. Ewig S, Torres A, Angeles Marcos M, Angrill J, Rano A, de Roux A, Mensa J, Martinez JA, de la Bellacasa JP, Bauer T. Factors associated with unknown aetiology in patients with community-acquired pneumonia. Eur Respir J. 2002;20:1254-62.

4. van Gageldonk-Lafeber AB, Heijnen ML, Bartelds Al, Peters MF, van der Plas SM, Wilbrink B. A case-control study of acute respiratory tract infection in general practice patients in the Netherlands. Clin Infect Dis. 2005;41:490-7.

5. Miao Q, Ma Y, Wang Q, Pan J, Zhang Y, Jin W, Yao Y, Su Y, Huang Y, Wang M, Li B, Li H, Zhou C, Li C, Ye M, Xu X, Li Y, Hu B. Microbiological diagnostic performance of metagenomic next-generation sequencing when applied to clinical Practice. Clin Infect Dis. 2018:67:S231-40.

6. Grumaz S, Stevens P, Grumaz C, Decker SO, Weigand MA, Hofer S, Brenner T, von Haeseler A, Sohn K. Next-generation sequencing diagnostics of bacteremia in septic patients. Genome Med. 2016;8:73

7. Lecuit $M$, Eloit $M$. The diagnosis of infectious diseases by whole genome next generation sequencing: a new era is opening. Front Cell Infect Microbiol. 2014:4:25.

8. Lefterova MI, Suarez CJ, Banaei N, Pinsky BA. Next-generation sequencing for infectious disease diagnosis and management: a report of the Association for Molecular Pathology. J Mol Diagn. 2015;17:623-34.

9. Khare R, Espy MJ, Cebelinski E, Boxrud D, Sloan LM, Cunningham SA, Pritt BS, Patel R, Binnicker MJ. Comparative evaluation of two commercial multiplex panels for detection of gastrointestinal pathogens by use of clinical stool specimens. J Clin Microbiol. 2014;52:3667-73.

10. Leber AL, Everhart K, Balada-Llasat JM, Cullison J, Daly J, Holt S, Lephart P, Salimnia H, Schreckenberger PC, DesJarlais S, Reed SL, Chapin KC, LeBlanc L, Johnson JK, Soliven NL, Carroll KC, Miller JA, Dien Bard J, Mestas J, Bankowski M, Enomoto T, Hemmert AC, Bourzac KM. Multicenter evaluation of BioFire FilmArray meningitis/encephalitis panel for detection of Bacteria, viruses, and yeast in cerebrospinal fluid specimens. J Clin Microbiol. 2016;54: 2251-61.

11. Ruggiero P, McMillen T, Tang YW, Babady NE. Evaluation of the BioFire FilmArray respiratory panel and the GenMark eSensor respiratory viral panel on lower respiratory tract specimens. J Clin Microbiol. 2014;52:288-90.

12. Tang YW, Gonsalves S, Sun JY, Stiles J, Gilhuley KA, Mikhlina A, Dunbar SA, Babady NE, Zhang $\mathrm{H}$. Clinical evaluation of the Luminex NxTAG respiratory pathogen panel. J Clin Microbiol. 2016;54:1912-4.

13. Chiu CY, Miller SA. Clinical metagenomics. Nat Rev Genet. 2019;20:341-55.

14. Moustafa A, Xie C, Kirkness E, Biggs W, Wong E, Turpaz Y, Bloom K, Delwart E, Nelson KE, Venter JC, Telenti A. The blood DNA virome in 8,000 humans. PLoS Pathog. 2017;13:e1006292.

15. Rascovan N, Duraisamy R, Desnues C. Metagenomics and the human Virome in asymptomatic individuals. Annu Rev Microbiol. 2016:70:125-41.

16. Somasekar S, Lee D, Rule J, Naccache SN, Stone M, Busch MP, Sanders C, Lee WM, Chiu CY. Viral surveillance in serum samples from patients with acute liver failure by metagenomic next-generation sequencing. Clin Infect Dis. 2017:65:1477-85.

17. Li H, Gao H, Meng H, Wang Q, Li S, Chen H, Li Y, Wang H. Detection of pulmonary infectious pathogens from lung biopsy tissues by metagenomic next-generation sequencing. Front Cell Infect Microbiol. 2018;8:205.

18. Houldcroft CJ, Beale MA, Breuer J. Clinical and biological insights from viral genome sequencing. Nat Rev Microbiol. 2017;15:183-92.

19. Schlaberg R, Queen K, Simmon K, Tardif K, Stockmann C, Flygare S, Kennedy B, Voelkerding K, Bramley A, Zhang J, Eilbeck K, Yandell M, Jain S, Pavia AT, Tong S, Ampofo K. Viral pathogen detection by Metagenomics and Panviral group polymerase chain reaction in children with pneumonia lacking identifiable etiology. J Infect Dis. 2017;215:1407-15.

20. Wilson MR, Naccache SN, Samayoa E, Biagtan M, Bashir H, Yu G, Salamat SM Somasekar S, Federman S, Miller S, Sokolic R, Garabedian E, Candotti F, Buckley RH, Reed KD, Meyer TL, Seroogy CM, Galloway R, Henderson SL, Gern JE, DeRisi JL, Chiu CY. Actionable diagnosis of neuroleptospirosis by next-generation sequencing. N Engl J Med. 2014;370:2408-17.

21. Rhodes J, Hyder JA, Peruski LF, Fisher C, Jorakate P, Kaewpan A, Dejsirilert S, Thamthitiwat S, Olsen SJ, Dowell SF, Chantra S, Tanwisaid K, Maloney SA, Baggett HC. Antibiotic use in Thailand: quantifying impact on blood culture yield and estimates of pneumococcal bacteremia incidence. Am J Trop Med Hyg. 2010;83:301-6.

22. Gosiewski T, Ludwig-Galezowska AH, Huminska K, Sroka-Oleksiak A, Radkowski P, Salamon D, Wojciechowicz J, Kus-Slowinska M, Bulanda M, 
Wolkow PP. Comprehensive detection and identification of bacterial DNA in the blood of patients with sepsis and healthy volunteers using nextgeneration sequencing method - the observation of DNAemia. Eur J Clin Microbiol Infect Dis. 2017;36:329-36.

23. Guo LY, Feng WY, Guo X, Liu B, Liu G, Dong J. The advantages of nextgeneration sequencing technology in the detection of different sources of abscess. J Inf Secur. 2019;78:75-86.

24. Fan S, Ren H, Wei Y, Mao C, Ma Z, Zhang L, Wang L, Ge Y, Li T, Cui L, Wu H, Guan H. Next-generation sequencing of the cerebrospinal fluid in the diagnosis of neurobrucellosis. Int J Infect Dis. 2018;67:20-4.

25. Hu Z, Weng X, Xu C, Lin Y, Cheng C, Wei H, Chen W. Metagenomic nextgeneration sequencing as a diagnostic tool for toxoplasmic encephalitis. Ann Clin Microbiol Antimicrob. 2018;17:45.

26. Ai JW, Li Y, Cheng Q, Cui P, Wu HL, Xu B, Zhang WH. Diagnosis of local hepatic tuberculosis through next-generation sequencing: smarter, faster and better. Clin Res Hepatol Gastroenterol. 2018;42:178-81.

27. Du B, Tao Y, Ma J, Weng X, Gong Y, Lin Y, Shen N, Mo X, Cao Q Identification of sparganosis based on next-generation sequencing. Infect Genet Evol. 2018;66:256-61.

28. Blauwkamp TA, Thair S, Rosen MJ, Blair L, Lindner MS, Vilfan ID, Kawli T, Christians FC, Venkatasubrahmanyam S, Wall GD, Cheung A, Rogers ZN, Meshulam-Simon G, Huijse L, Balakrishnan S, Quinn JV, Hollemon D, Hong DK, Vaughn ML, Kertesz M, Bercovici S, Wilber JC, Yang S. Analytical and clinical validation of a microbial cell-free DNA sequencing test for infectious disease. Nat Microbiol. 2019;4:663-74.

29. Deurenberg RH, Bathoorn E, Chlebowicz MA, Couto N, Ferdous M, GarciaCobos S, Kooistra-Smid AM, Raangs EC, Rosema S, Veloo AC, Zhou K, Friedrich AW, Rossen JW. Application of next generation sequencing in clinical microbiology and infection prevention. J Biotechnol. 2017;243:16-24.

30. Gargis AS, Kalman L, Lubin IM. Assuring the quality of next-generation sequencing in clinical microbiology and public health laboratories. J Clin Microbiol. 2016;54:2857-65.

31. Miller S, Naccache SN, Samayoa E, Messacar K, Arevalo S, Federman S, Stryke D, Pham E, Fung B, Bolosky WJ, Ingebrigtsen D, Lorizio W, Paff SM, Leake JA, Pesano R, DeBiasi R, Dominguez S, Chiu CY. Laboratory validation of a clinical metagenomic sequencing assay for pathogen detection in cerebrospinal fluid. Genome Res. 2019;29:831-42.

\section{Publisher's Note}

Springer Nature remains neutral with regard to jurisdictional claims in published maps and institutional affiliations.

Ready to submit your research? Choose BMC and benefit from:

- fast, convenient online submission

- thorough peer review by experienced researchers in your field

- rapid publication on acceptance

- support for research data, including large and complex data types

- gold Open Access which fosters wider collaboration and increased citations

- maximum visibility for your research: over $100 \mathrm{M}$ website views per year

At $\mathrm{BMC}$, research is always in progress.

Learn more biomedcentral.com/submissions 